\title{
SIMPLIFIED TECHNICAL ENGLISH IN MIRCE MECHANICS
}

\author{
Orlando Chiarello* \\ Secondo Mona S.p.A., Italy \\ Dr Jezdimir Knežević \\ MIRCE Akademy, Woodbury Park, Exeter, United Kingdom
}

The second Axiom of Mirce Mechanics, states, "The probability of faulty execution of any maintenance task is greater than zero". Analysis of maintenance processes clearly shows that ineffective communication between system designers and maintenance personnel, through maintenance documentation, is a well-recognised contributor to the occurrence of faulty maintenance task, which in turn could have a significant impact on reliability, availability, safety, cost and effectiveness of technical systems. This paper addresses the lack of understanding of maintenance manuals, written in English, by $80 \%$ of the global maintenance personnel whose native language is not English. The majority of them have knowledge of English that is rather limited and are easily confused by complex sentence structures and by the number of meanings and synonyms that English words may have. Significant improvements in the direction of effective communication have been achieved by the creation and use of Simplified Technical English, the benefit of which is presented in this paper.

Keywords: Mirce mechanics, Maintenance errors, Maintenance documentation, Simplified technical english, Technical authors training

\section{INTRODUCTION}

Mirce Mechanics: is a scientific theory of the motion of observable functionability phenomena through the life of technical systems. Its axioms, mathematical formulas, rules and methods enable accurate predictions of a system's measurable functionability performance characteristics like reliability, availability and others to be made with probabilistic regularity [08].

According to Mirce Mechanics the motion of functionability through negative functionability state is driven by the execution of maintenance tasks, among other factors. Maintenance tasks typically include removal, installation, servicing, rigging, inspection and other scheduled maintenance. The execution of any maintenance task involves the possibility of human error. Human error in aircraft maintenance is the consequence of a complex interaction of many factors including system and maintenance task design, maintenance personnel and other resources, maintenance organisation, and the physical environment in which the maintenance occurs.

Hence, to assess the impact of human errors on successful execution of maintenance tasks, analysis of over tens of thousands of maintenance tasks, in defence, aerospace, transportation, motorsport, nuclear, communication and other industries, had been studied at the MIRCE Akademy. It has lead to the formulation of the second Axiom of Mirce Mechanics, which states, "The probability of faulty execution of any maintenance task is greater than zero". [09] As it has a profound impact on all aspects of the in-service life on any maintainable technical system several research studies have been performed by the Master and Doctoral students of the MIRCE Akademy with aim to understand the physical mechanisms that caused their occurrences.

The human constituents of a maintenance process, either as decision maker or as a task executor, bear the ultimate responsibility for recognising, interpreting, compensating for, and correcting or mitigating the consequences of deficiencies and faults of a maintenance process. Thus "human error" and "judgement error" are terms found frequently in reports on malfunctioning of technical systems. For example, according to a Pratt \& Whitney survey the major causes for the 120 in-flight engine shutdowns on Boeing 747 aircraft's, due to human errors 
in maintenance, were mainly due to:

- Incomplete installation (33\%)

- Damaged on installation (14.5\%)

- Improper installation (11\%)

- Equipment not installed or missing (11\%)

- Foreign object damage (6.5\%)

- Improper fault isolation, inspection (6\%)

- Equipment not activated or deactivated (4\%)

Some of the other related causes are:

- Complex maintenance related tasks

- Time pressure for delivering the aircraft

- Fatigue of the maintenance personnel

- Maintenance procedures not followed accordingly

- Usage of outdated maintenance manuals

However, as statistics does not study the causes of statistical behaviour, full understanding of the human errors in maintenance is only possible by understanding physical causes and mechanisms that lead to the occurrence of maintenance faults during the maintenance process.

Analysis of maintenance processes clearly shown that ineffective communication between system designers and maintenance personnel, through maintenance documentation, is a wellrecognised contributor to the occurrence of faulty maintenance task, which in turn could have a significant impact on reliability, availability, safety, cost and effectiveness of a system [07].

For example, in the article [10] it was reported that in a given service bulletin a certain maintenance procedure was "proscribed". The technician reading this bulletin concluded to proceed and perform that task as it was "prescribed". Clearly this is an excellent example of misunderstanding of English word "proscribed", which means "prohibited" with the English word "prescribed" which means "defined".

Consequently, the main objective of this paper was to address the lack of understanding of maintenance manuals, written in English, in relation to the second Axiom of Mirce Mechanics.

\section{THE FUNCTIONS OF THE MAINTENANCE DOCUMENTATION}

The maintenance documentation used in maintenance of technical systems has the following three functions:

- To support correct execution of a maintenance task. It is a procedural document that guides maintenance personnel trough a sequence of instructions in the task execution.
Comprehensible and accurate documentation is a vital part of the successful execution of a maintenance task.

- To support maintenance training. In well-organised and structured organizations maintenance documentation is used during the training process. However, in some organisations maintenance documentation is discovered after the unsuccessful completion of a task.

- To support a legal process, when necessary. For example, in aviation industry the use of maintenance documentation is a legal obligation. It constitutes a proof that a maintenance task has been executed in accordance with the related instructions. Thereby, the maintenance documentation is a mean of certifying safety and security of the aircraft systems after the completion of a maintenance task. Indeed, the maintainer must sign in the documentation used at the end of the maintenance operation. The signature has an important legal role. For third party maintenance organizations, it constitutes a protection in case of a conflict with the customer after the delivery of the aircraft.

\section{ENGLISH AS THE GLOBAL LANGUAGE}

With the development of "computerized" technical reality, English became the international language mostly used by communities, organizations and industries for promoting their business and defining their products. However, it is not the native language of the majority of the people involved. Hence, potentials for misunderstandings and miscommunications are endless and continuously present.

The first solution to this problem was proposed by Professor Charles K. Ogden in the Thirties who created the Basic English [06] that consisted of a set of simple grammar rules and a restricted vocabulary of 850 words. It was the first real attempt to "give everyone a second or international language" for business and education in every country, with the primary aim of creating more communication among people. Therefore, Basic English can be considered as a pioneer controlled language. From there, some other controlled languages were developed, each of them dedicated to different fields of application. In the Seventies, Caterpillar created the Caterpillar Fundamental English [6] to make the operating and maintenance instructions of their products easily understood by their customers and users. 
In the Nineties, the President of the United States introduced the use of Plain Language in all government regulations, the Great Britain Government, and other English-speaking Countries used the same approach. The new South African Constitution of 1996 is perhaps the first in the world written in scrupulous respect of the principles of Plain Language.

\section{ASD SIMPLIFIED TECHNICAL ENGLISH}

English became also the language of industrial and technological domains, especially aviation and military industries where the need of sharing a common code for operation, maintenance and logistics support was essential to guarantee, other than the correct understanding of the procedures, the flight safety and the human life. A standard was created to regulate technical writing: the ASD Simplified Technical English, Specification ASD-STE100 (STE) [01].

The STE project (formerly known as AECMA Simplified English) started in 1979 with a request made by the Association of European Airlines, which approached AECMA (the European Association of Aerospace Industries) to investigate a possible form of controlled English to be used by all manufacturers in their maintenance manuals. After researching several types of controlled languages that existed in other industries, AECMA decided to produce its own controlled English and, in 1983, set up a project group, under the leadership of Fokker. The project was not limited to the European industry and the American Aerospace industry, through the AIA (Aerospace Industries Association of America), was invited to participate, especially as some American companies had already done some standardization along the same lines.

AECMA Simplified English was first released in 1986 as a guide. Soon, it was included as a requirement in the major international specifications for writing maintenance manuals such as ATA100 (now ATA Spec i2200) [09] and S1000D [03]. In 2004, AECMA became ASD, "The AeroSpace and Defence Industries Association of Europe" and the Simplified English guide became an official specification, ASD-STE100, with the word "technical" added to its name. Although today the STE structure is stable and consolidated, the language has to be kept in line with the technology evolution and amended on the continuous and important feedback from the users. Today, the success of STE is such that other in- dustries want to use it beyond its intended purpose of aviation maintenance documentation.

\section{PRINCIPLES AND STRUCTURE OF STE}

The STE specification provides a set of writing rules and a dictionary of controlled vocabulary. The writing rules (approximately 60) cover aspects of grammar and style; the dictionary (approximately 860 approved words) specifies the general words that can be used. These words were chosen for their simplicity and ease of recognition. In general, there is only one word for one meaning, and one part of speech for one word.

The STE specification consists of two parts, namely:

Part 1: Writing Rules, which specifies restrictions on grammar and style usage. For example, they require writers to:

- Use the approved words in the Dictionary, and the relevant Technical Names and Technical Verbs

- Use consistent language and spelling

- Avoid slang and jargon

- Make instructions as specific as possible

- Restrict the length of noun clusters to no more than 3 words

- Use articles when appropriate

- Use simple verb tenses (past, present, and future)

- Use active voice and not passive

- Not use -ing participles or gerunds

- Keep to one topic per sentence

- Use vertical lists for complex texts

- Keep sentence length as short as possible (20 words maximum for procedural sentences, or 25 words maximum for descriptive sentences)

- Write sequential steps as separate sentences

- Write only one instructions per sentence

- Use the imperative (command) verb in procedures

- Use paragraphs to show the reader the logic of text

- Restrict paragraphs length to no more than 6 sentences

- Identify and write warnings and cautions correctly

- Use notes to give information and not commands

- Use punctuation correctly 
- Do different constructions when it is not possible to replace an unapproved word with an approved word

Part 2: Dictionary, which includes entries of both approved and unapproved words. The approved words can only be used in their specified meaning. For example, the word "close" (verb) can only be used in one of two approved meanings:

1. To move together or to move to a position that stops or prevents materials from going in or out.

2. To operate a circuit breaker to make an electrical circuit.

The verb can be used to express "close a door" or "close a circuit", but it cannot be used in other senses (for example "close the meeting" or "close a business"). The adjective "close" appears in the Dictionary as an unapproved word with the suggested approved alternative "near" (as a preposition). So STE does not allow "do not go close to the landing gear", but it does allow "do not go near the landing gear".

Besides the specified general vocabulary, STE accepts the use of company-specific or projectoriented technical words (referred to in STE as Technical Names and Technical Verbs), if they fit into one of their categories listed in the specification. Section 1, Words, gives explicit guidelines for using technical terms and verbs that writers need. For example, nouns such as "overhead panel", "grease", "propeller", or the verbs " ream", and "drill" are not listed in the Dictionary, but they qualify as approved terms under the guidelines listed in Part 1, Section 1 (specifically, Writing Rules 1.5 and 1.13).

\section{STE AND TRANSLATION}

Helping the translation process was a primary goal of the controlled languages and STE is no exception. As said before, the Simplified English project officially started on June 30,1983 , with the "First Ratification Meeting for AECMA Simplified English", held at Fokker, Amsterdam. This significant statement is included in the minutes of that meeting:

"Simplified English should be seen as a code that uses English words for its symbols. The users of the texts learn to recognize the "code symbols" (words) that tell them what to do. The users do not learn how to speak or write English. They do not even learn "everyday" English, but only SE. But at the same time, SE must still meet the de- mand of being an idiomatic version of English. ... This "code" aspect of SE makes it very suitable for machine translation". [02]

The use of STE throughout the years indicates that translation from STE texts is not necessary in aviation if the readers have a basic knowledge of English but it may be necessary if the readers do not have that knowledge. However, outside aviation a translation could always be necessary. In this case, if the "source text" is English and correctly written in STE, the translation, especially Machine Translation, can be dramatically helped by the principle of "one word = one meaning". The translation will be more and more accurate if the machine is "guided" to replicate in its translation the "assigned meaning" given to the STE words. This does not necessarily require subsets of STE translated in other languages but the availability of a "mirror" controlled language based on STE would greatly help the translation process. A "mirror" controlled language may use the same structure of the STE writing rules and dictionary suitably adapted to a specific language. A significant attempt was made in the past in France (Rationalized French) with exceptionally good results and enormous benefit to translation. Other attempts were made with other languages and some are in process.

Having as a reference a consolidated and internationally recognized standard is very important in translation. It could be ASD-STE100 or another standard but the starting text should be correct, simple and understandable. Sometimes people are confused by the instructions. For example, they may not be able to set their TV, mobile phone or video camera, because the texts in the user's manuals are not translated correctly into their language. These manuals are clear examples in which the starting texts - although understandable in the original starting language - can be misinterpreted by the translators and the resulting translation may lead to something incorrect or meaningless. The common "code" (i.e. the Standard) is essential to the effective translation and communication in general.

\section{STE AUTHORING TOOLS}

The primary objective of STE is the creation of simplified texts for the readers. However, STE is not a simplified version of English for the writers. Writing correctly in STE is not an easy task as it requires a good command of the English language together with a good knowledge of the 
matter of the writing. This combination is without a doubt the key to writing successfully in STE. Authors who would like to write proficiently and correctly in STE must have as the only point of reference the STE specification itself. There is nothing that can replace it. The use of manipulated versions of the STE specification, partial use of the specification or deviations from its writing rules and vocabulary will diminish the accuracy of STE and create confusion among its users.

To assist users and potential users of STE, there are on the market software products that support STE. The basic question is: "Do we need a software product to write in STE correctly?" Well, the answer is "no" simply because software does not think in place of authors and does not replace the STE specification.

STE checkers should only be seen as aids for those authors having a good knowledge of STE. None of these checkers write STE text for authors, nor can they convert non-STE text into STE. Although STE checkers can be helpful with highlighting non-STE terms and incorrectly written STE text, they are not fool-proof. We have cases in which STE checkers parse STE texts correctly (no errors flagged) even if these texts do not obey the Standard English Grammar! Authoring tools must be used with discernment and if authors rely blindly on what checkers tell them, they are likely to write rubbish. The authors are the ones who know, think and control. Only the authors are the ones who must decide whether what a tool has told them is correct or not.

\section{TRAINING PROGRAMME IN STE AT THE MIRCE AKADEMY}

Effective maintenance communication is very important and sharing a common "code" is essential. In the present global and complex scenario, Standards are playing a key role for the purpose. In the same way that XML regulates the formatting of texts for electronic release, there is strong need to regulate the writing itself. The controlled languages, as internationally recognized standards, can serve the scope in certain instances. The use of controlled languages in writing and translation does not diminish the everyday languages but makes the messages and texts easily understandable to everyone. The misconception about controlled languages, reluctance and resistance to use them are often derived by the fact that it is thought they could limit the authors. In reality, nobody will try to translate poems or literature books into STE or similar, and it is necessary to keep literature material well separated from general communication and technical domains. Plain Language, Plain English, Simplified Technical English etc. should be seen as vehicles and tools for improving clarity and hit the target of effective communication. Without standards, there will be a serious risk of an uncontrolled "jungle".

Consequently, in collaboration with Secondo Mona, the MIRCE Akademy offers the opportunity for learning the needs for and principles of effective technical communication in general and its in-depth applications to maintenance. It is based on the philosophy and methods of ASD-STE100, which is created to prevent the misunderstanding of technical instructions that can lead to errors and accidents. An example of a 30-hour training course in STE offered by the Akademy is given in the Appendix.

\section{CONCLUSIONS}

Analysis of maintenance tasks required during the in-service life of technical systems, preformed under the auspices of Mirce Mechanics, clearly showed that ineffective communication between system designers and maintenance personnel, through maintenance documentation, is wellrecognised contributor to the occurrence of faulty maintenance task, which in turn could have a significant impact on reliability, availability, safety, cost and effectiveness of technical systems.

Although, English is the language of industrial and technological domains, especially aviation and military industries, where the need of sharing a common code for operation, maintenance and logistics support is accepted, for $80 \%$ of global population it is not native language. Many of them have knowledge of English that is rather limited and are easily confused by complex sentence structures and by the number of meanings and synonyms that English words may have.

Significant improvements in the direction of effective technical communication have been achieved by the creation of controlled languages, and a standard that regulates technical writing: the ASD Simplified Technical English, ASDSTE100 (STE). STE, as described in this paper and as a consolidated international standard, can be seen as the gate to successful and effective technical communication. As previously remarked, today the success of STE is such that other industries want to use it beyond its intend 
ed purpose of aviation maintenance documentation. STE interest is also growing within the Academic world. For its principles and accuracy it is a model-writing standard for other domains and industries like medical, oil, high-tech, IT, automotive, and many others. A recent study on STE shows that only $3 \%$ of the current content is specific to aviation, the remaining $97 \%$ is applicable in all contexts, without any need of adaptation.

The same study shows that, during the writing process, authors tend to focus mainly on the Dictionary and not on the Writing Rules, which are equally important. Simply giving authors a copy of the STE specification is probably the best way to discourage them from learning about STE, and applying it correctly. It is important for authors to fully understand the STE writing rules, and to think about what they are writing. Therefore, STE training is the first essential step for a technical author to be able to apply STE correctly. The STE training programme for technical writers, conducted at the MIRCE Akademy, has been briefly discussed and presented.

\section{REFERENCES}

1) ASD - Aerospace and Defence Industries Associations of Europe - "ASD Simplified Technical English, Specification ASD-STE100", 1986 - 2013.

2) ASD - Aerospace and Defence Industries Associations of Europe - "First Ratification Meeting for AECMA Simplified English", 1983 - 2013.

3) ASD - Aerospace and Defence Industries Associations of Europe - "S1000D - International specification for technical publications utilizing a common source database", 1989 - 2009

4) ATA, Air Transport Association of America - "ATA iSpec 2200, Information Standards for Aviation Maintenance", 1956 and subsequent dates of revisions and issues

5) Charles A. Verbeke, "Caterpillar Fundamental English", Training and Development Journal, 27, 2, 36-40, Feb 1973.

6) Charles K. Ogden, "BASIC English - Ageneral introduction with rules and grammar", London, Kegan Paul, Trench, Trubner \& Co. Ltd., 1930 and subsequent editions.

7) Chiarello, O., "Impact of Accuracy of Technical Communication on the Motion of Functionability", Proceedings of the 1st World Congress of Mirce Mechanics, 28-30 May 2012, Exeter, UK

8) Knezevic, J. (2012): Time to Choose Between Scientific and Administrative Approach to Reli- ability, Journal of Applied Engineering Science, Vol.10, No. 3, pp. 167-173

9) Knezevic, J., "Quality of Maintenance - Mirce Mechanics axiom", Journal of Quality in Maintenance Engineering, Vol. 18, No 2, 2012, pp 216-226, Emerald Group Publishing Ltd. UK

10) Werfelman, L. "Simplifying the Technicalities", Flight Safety Foundation, AeroSafety World, August 2007.

Paper sent to revison: 05.02.2013.

Paper ready for publication: 05.03.2013.

Appendix: Training Course in Effective Maintenance Communication

\section{The Program}

Day one:

Operational Reliability, Cost Effectiveness of Technical Systems, Mirce Mechanics Principles Maintenance induced failures, types and categories Impact of Maintenance Communication on Inservice Reliability, Cost and Effectiveness

Introduction to ASD-STE100

- What is Simplified Technical English?

- Why do we need a controlled language? Other controlled languages

- History, background and philosophy of STE

- Who uses STE? Is it only for aviation and maintenance?

- The ASD-STE100 Specification: Part 1 Writing Rules

- How to use the Dictionary

- Overall overview of the Writing Rules

- Detailed tutorial of the Writing Rules

- Section 1 - Words

- Section 2 - Noun Phrases

Day two:

Writing Rules

- Section 3 - Verbs

- Section 4 - Sentences

- Section 5 - Procedures

- Section 6 - Descriptive Writing

- Section 7 - Warnings, Cautions and Notes

- Section 8 - Punctuation and Word Count

- Section 9 - Writing Practices

- Practical exercises are given after the tutorial of each section

Day Three

Final review of the Specification

Practical texts (also proposed by the participants)

Final Test and its correction

Question time, final discussion and course assessment Certificate Award Ceremony 